\title{
O impacto da depressão para as interações sociais de universitários
}

\section{The impact of depression for social interactions of college students}

\section{El impacto de la depresión para las interacciones sociales para los estudiantes universitarios}

\section{Alessandra Turini Bolsoni-Silva*}

Universidade Estadual Paulista - UNESP, Bauru, São Paulo, Brasil

\author{
Bárbara Trevizan Guerra** \\ Universidade Estadual Paulista - UNESP, Bauru, São Paulo, Brasil
}

\begin{abstract}
RESUMO
Estudos têm demonstrado que condições aversivas na universidade podem ter grande influência no aproveitamento acadêmico e no desenvolvimento de transtornos psiquiátricos, como a depressão. Nesse contexto universitário, as interações sociais têm sido alvo de investigações ao longo dos anos, mas são escassas publicações referentes a habilidades sociais e saúde mental. Assim, o presente artigo comparou um grupo de estudantes universitários clínico com um não clínico para depressão em relação às consequências e sentimentos que diferentes respostas de habilidades sociais podem ter em interações sociais com diversos interlocutores. Participaram 128 estudantes, sendo 64 clínicos para depressão e 64 não clínicos. Foram aplicados os Questionários de Avaliação de Comportamentos e Contextos para Universitários (QHC - Universitários), Inventário de Fobia Social (Mini-Spin), Inventário de Depressão de Beck (BDI) e Entrevista clínica estruturada para o DSM-IV (SCID-I). Os dados foram analisados a partir do teste $t$ de Student. Os resultados atestam que os universitários com depressão apresentam importante déficit quanto ao repertório de habilidades sociais independente das interações sociais estabelecidas com diferentes interlocutores, seja com familiares, amigos, colegas de república ou namorados.
\end{abstract}

Palavras-chave: depressão, universitários, habilidades sociais, saúde mental.

\begin{abstract}
Studies have shown that aversive conditions at the university may have great influence on academic achievement and the development of psychiatric disorders such as depression. In university context, social relations have been investigated over the years, but are scarce publications relating mental health and social skills. Thus, this paper compared a group of college students with a clinical depression with a group without clinical depression for the consequences and feelings that differents responses of social skills may have in social interactions with various interlocutors. A total
\end{abstract}


of 128 students participated, 64 for clinical depression and 64 non-clinical. Questionário de Avaliação de Comportamentos e Contextos para Universitários - QHC - Universitários, Inventário de Fobia Social (Mini-Spin), Inventário de Depressão de Beck (BDI) and Entrevista clínica estruturada para o DSM-IV (SCID-I) were applied. The data were analyzed by test $t$ of Student. The results show that students with depression have a great difficulty about the social skills regardless of which require more or less assertion and general way for all social interactions, either family, friends, roommate and partner.

Keywords: depression, academics, social skills, mental health.

\section{RESÚMEN}

Los estudios han demostrado que las condiciones aversivas de la universidad pueden tener gran influencia en el rendimiento académico y el desarrollo de trastornos psiquiátricos, como la depresión. En el ámbito universitario, las relaciones sociales se han investigado en los últimos años, pero son escasas las publicaciones relacionadas con la salud mental y las habilidades sociales. Por lo tanto, em este trabajo se comparó un grupo de estudiantes universitarios con depresión clínica con un no-clinico para las consecuencias de las diferentes respuestas y sentimientos que pueden tener habilidades sociales en las interacciones sociales con diversos interlocutores. Un total de 128 estudiantes, 64 para la depresión clínica y no clínica 64 participaron. Questionário de Avaliação de Comportamentos e Contextos para Universitários - QHC - Universitários, Inventário de Fobia Social (MiniSpin), Inventário de Depressão de Beck (BDI) y Entrevista clínica estruturada para o DSM-IV (SCID-I) se aplicaron. Los datos se analizaron mediante el test $t$ de Student. Los resultados muestran que los estudiantes con depresión tienen una gran dificultad sobre las habilidades sociales independientemente de que requieren más o menos la afirmación y de manera general para todas las interacciones sociales, ya sea con la familia, amigos, coelgas de la república o namorados.

Palabras-clave: depresión, universitarios, habilidades sociales, salud mental.

\section{Introdução}

A universidade é um contexto de interações sociais diversas e exige do universitário muitas habilidades interpessoais e acadêmicas. Tais demandas podem agravar problemas de saúde mental já existentes ou podem aumentar a probabilidade de ocorrerem.

Por desafios podem ser elencados: estudar diferentemente da forma como se preparou para o vestibular, lidar com autoridade (professor), falar em público (por exemplo, apresentar seminários), fazer novas amizades, morar com outras pessoas, por vezes desconhecidas (repúblicas), negociar divisão de tarefas (repúblicas), cuidar de si mesmo e dos próprios pertences, ficar longe da família, amigos e namorado(a), administrar renda e trabalhar para se sustentar. Todas essas novas exigências irão requerer repertórios diferenciados dos estudantes e dependendo da história de vida (Facundes \& Ludermir, 2005; Ribeiro \& Bolsoni-Silva, 2011) e de quanto estiverem 
instrumentalizados para tais desempenhos, irão lidar com essas demandas de modo mais eficiente e adaptado.

A depressão, segundo o DSM-IV-TR (APA, 2000) é um transtorno mental no qual há significativa alteração do humor ou afeto, está frequentemente associada à incapacitação funcional e prejuízo na qualidade de vida, em razão de sua sintomatologia caracterizada por tristeza, apatia, redução da energia, perda de interesse nas atividades, diminuição da capacidade de concentração, fadiga, alterações no apetite e no sono, culpabilidade, diminuição da autoestima e da autoconfiança.

Conforme Ferster (1973), a pessoa depressiva se engaja em alta frequência em comportamentos de evitação e esquiva de estímulos aversivos, geralmente na forma de reclamar, de fazer pedidos. Sabese que ocorre também a diminuição da frequência de comportamentos reforçados positivamente. A pessoa repetidamente pode, por exemplo, se engajar em dizer como está mal, chorar, falar de suicídio e reclamar de fadiga e doença.

Vasconcellos, Rocha e Maciel (2010) afirmam que, para a Análise do Comportamento, a depressão deve ser entendida como um padrão complexo de interação com o ambiente, sendo necessária para o seu entendimento uma análise funcional do mesmo, ou em outras palavras, para além da topografia de resposta é necessário descrever as contingências em operação.

Para a realização da análise funcional é preciso considerar que o indivíduo interage com o ambiente a partir de estímulos antecedentes e consequentes, sendo que esses últimos vão influenciar a emissão dos comportamentos futuros, aumentando ou diminuindo sua frequência (Tourinho, 1999). É necessário ainda identificar classes de respostas, de estímulos antecedentes e consequentes, compreendendo não apenas a função da resposta excessiva ou deficitária, mas também as interdependências comportamentais (Goldiamond, 1974/2002).

Como subproduto das interações sociais há a presença de sentimentos positivos, associados a consequências reforçadoras, e sentimentos negativos que surgem contingentes às estimulações aversivas (Abib, 2001). Segundo Delitti e Meyer (1998) tais eventos privados (pensamentos, sentimentos e sonhos) podem ser passíveis de coleta de dados e analisados, pois trazem elementos relevantes da história de aprendizagem do indivíduo.

Em relação às contingências aversivas, vivências acadêmicas que não garantem uma boa qualidade de vida podem tornar-se experiências estressantes, podendo tanto influenciar no aparecimento de transtornos depressivos, quanto afetar o rendimento acadêmico (Backer, 2003; Ciarrochi, Deane, \& Anderson, 2002; Cole, Lazarick, \& Howard, 1986; Furtado, Falcone, \& Clark, 2003) e o abandono escolar (McGaha \& Fitzpatrick, 2005). 
Considerando-se o transtorno depressivo, o estudo das habilidades sociais (HS) na interface com a saúde mental, seja na população geral, seja entre universitários, torna-se importante por favorecer a identificação e consequente promoção da saúde, evitando comprometimentos na vida profissional, amorosa e de lazer. BolsoniSilva (2002) investiga a possibilidade de estudo de HS à luz da Análise do Comportamento, pois esta permite a análise de eventos privados, o estudo de comportamentos respondentes e operantes (modelo de assertividade), aponta para a plasticidade do comportamento, e também para a possibilidade de aprendizagem por observação, claramente possível (Catania, 1998/1999); todavia tais operantes só se mantêm no repertório se produzirem consequências reforçadoras. A autora ainda considera que as habilidades sociais podem ser classificadas como comportamentos operantes comportamentos que são mantidos pelas suas consequências e, portanto, passíveis de análise funcional, avaliação e intervenção.

As HS de universitários têm sido objeto de estudo, destacando-se, enquanto temas e dificuldades, o lidar com relacionamentos amorosos (Del Prette \& Del Prette, 2003; Del Prette et al., 2004; Boas, Silveira, \& Bolsoni-Silva, 2005; Del Prette, Del Prette, \& Barreto, 2006), com familiares (Bandeira \& Quaglia, 2005) e demandas acadêmicas (Teixeira, Dias, Wothich \& Oliveira, 2008). Enquanto comportamentos podem ser mencionados: expressar sentimento positivo (Del Prette et al., 2004; Bandeira, Rocha, Freitas, Del Prette \& Del Prette, 2006; Bolsoni-Silva, Loureiro, Rosa \& Oliveira, 2010; Ribeiro \& Bolsoni-Silva, 2011), negativo (Bandeira et al., 2006; Bolsoni-Silva, Loureiro, Rosa, \& Oliveira (2010); Ribeiro \& Bolsoni-Silva, 2011), opiniões (Bolsoni-Silva et al., 2010; Ribeiro \& Bolsoni-Silva, 2011), solicitar mudança de comportamento (Bandeira et al., 2006), comunicar-se (Bolsoni-Silva et al., 2010), resolver conflitos (Bolsoni-Silva et al., 2010), lidar com críticas (Ribeiro \& Bolsoni-Silva, 2011) e falar em público (Ribeiro \& Bolsoni-Silva, 2011).

Em termos de comportamentos, sumarizando os estudos mencionados, conclui-se que o expressar sentimento positivo, solicitar mudança de comportamento, expressar sentimentos negativos, falar em público e lidar com críticas são os maiores déficits dos repertórios dos universitários. No entanto, os estudos acima citados não estavam preocupados em verificar a ocorrência dessas dificuldades na interface com problemas de saúde mental, como o caso da depressão.

Características sócio-demográficas também parecem influenciar o repertório de HS e condições de saúde mental. Cerchiari, Caetano e Faccenda (2005) verificaram que os alunos que tinham uma atividade remunerada apresentaram melhores indicadores de saúde mental. Os autores destacaram também que pertencer à maioria étnica, morar 
com a família, trabalhar com remuneração e ser casado podem ser fatores de proteção para a saúde do universitário. Adicionalmente, o sexo feminino parece estar mais predisposto a desenvolver transtornos na universidade (Adlaf, Gliksman, Demers, \& NewtonTaylor, 2001; Cerchiari et al., 2005; Neves \& Dalgalarrondo, 2007), bem como a procurar por serviço psicológico (Peres, Santos, \& Coelho, 2004).

$\mathrm{O}$ ano do curso e o tipo de curso também foram objeto de estudo, enquanto variáveis preditivas de saúde mental, tal como relatado a seguir. Cerchiari et al., (2005) encontraram maior prevalência de transtornos em estudantes dos quarto e quinto anos (Enfermagem e Ciência da Computação). Neves e Dalgalarrondo (2007) também identificaram alta prevalência de transtornos em estudantes da área de saúde $(56,3 \%)$, mas, no entanto, perde para a área de humanas e artes $(68,1 \%)$, e as áreas de ciências básicas, exatas e tecnológicas apresentaram baixa prevalência $(4,7 \%)$.

Outros fatores que podem contribuir para os transtornos mentais foram apresentados em uma pesquisa realizada por Fioretti, Rossoni, Borges, \& Miranda (2010), em que os dados indicaram que fatores presentes antes da graduação, tais como não receber apoio emocional necessário e dificuldades em tirar dúvidas, por timidez, podem ser fatores de risco para o desenvolvimento dos transtornos.

Dentre os estudos internacionais relativos a problemas de saúde mental e habilidades sociais de universitários, Edmondson, Conger e Conger (2007) encontraram que quanto maior o nervosismo e a tristeza, menor os escores de competência social e expressividade afetiva. Fitts, Sebby e Zlokovich (2009) examinaram se estilo de humor de 132 alunos universitários americanos, relacionando filiação, autorreforço (enhancing), comportamento agressivo e autodestrutivo e a percepção de competência social foram relacionados com timidez e isolamento social.

Esses estudos sugerem uma relação entre habilidades sociais e depressão, mas nota-se que as dimensões e repertórios foram restritos considerando os comportamentos indicados como dificuldades para os universitários, os quais foram mencionados previamente. Adicionalmente, pouco é investigado sobre o impacto das habilidades sociais nas interações sociais estabelecidas, sobretudo na interface com a saúde mental.

Fang, Young, Golshan, Moutier e Zisook (2010) investigaram a incidência de depressão entre estudantes de medicina nos Estados Unidos. Compararam estudantes que estavam no início e no final do curso e constataram que os estudantes do início do curso apresentavam maiores índices de depressão. Quanto às variáveis sócio-demográficas encontraram que os graduandos hispânicos e do sexo feminino apresentaram maiores índices de depressão, sendo que 
tal informação em relação ao sexo, foi confirmada por outras pesquisas, tais como a de Neves \& Dalgalarrondo (2007).

Corroborando com os estudos anteriores, Ozben (2013), que teve por objetivo investigar habilidades sociais, satisfação de vida e níveis de solidão em 525 universitários, concluiu que habilidades sociais e satisfação com a vida foram negativamente correlacionados com solidão e, por outro lado, habilidades sociais foram correlacionadas positivamente com satisfação de vida. Desta forma, tal pesquisa embasa a afirmação da relação inversamente proporcional existente entre habilidades sociais e transtornos mentais.

Ainda que os achados empíricos atestem para correlações entre habilidades sociais e depressão, faltam estudos que avaliem múltiplas classes de respostas de habilidades sociais e que avaliem as respostas, os sentimentos e os contextos sociais em que ocorrem.

Com base nessas considerações, a presente pesquisa compara as interações sociais de estudantes universitários com e sem indicação para depressão, descrevendo as consequências e os sentimentos que as habilidades sociais têm frente a diferentes interlocutores.

\section{Método}

\subsection{Aspectos éticos}

Este trabalho é parte de um projeto que prevê avaliação e intervenção junto a universitários cujo título é Análise das habilidades sociais de grupos universitários, o qual foi aprovado em 30 de agosto de 2007 pelo Comitê de Ética em Pesquisa da universidade em que está vinculado (processo no 1315/46/01/07). A coleta de dados foi iniciada em 2008, após a aprovação do referido Comitê de Ética.

\subsection{Participantes}

Participaram desse estudo 128 estudantes universitários (idade média de 21 anos, desvio padrão de 3,16$)$, graduandos de diferentes cursos de uma universidade pública do centro-oeste paulista, distribuídos em todos os anos dos cursos que frequentavam, sendo 64 clínicos para depressão e 64 não clínicos, sendo 24 homens clínicos e 24 homens não clínicos e 40 mulheres clínicas e 40 não clínicas. Quanto à distribuição por cursos têm-se: 24 universitários da área de exatas, 37 da área de humanas e três de biológicas, cujo critério de seleção da amostra está descrito em procedimentos.

\subsection{I nstrumentos}


Questionário de Avaliação de Comportamentos e Contextos para Universitários - QHC-Universitários (Bolsoni-Silva, 2011): é um questionário composto por questões que se referem à forma como o participante se comporta com relação a seus pais, amigos, namorado (a), entre outros, bem como os contextos em que ocorrem os comportamentos, como os interlocutores reagem a eles e os sentimentos dos universitários nessas ocasiões. O instrumento é constituído pelos seguintes tópicos: 1) Comunicação, 2) Expressividade (Sentimentos positivos, negativos e opiniões), 3) Críticas (fazer e receber críticas), 4) Falar em público (falar para público - conhecido e desconhecido, frequencia e dificuldade em apresentar seminários) e 5) Informações Adicionais (caso a pessoa deseje acrescentar algo que não foi abordado no questionário). Foi validado por Bolsoni-Silva (2011) com satisfatórios resultados de validade discriminativa, concorrente e de critério. No total o questionário apresenta 19 questões de frequência e 217 opções de resposta de conteúdo, cujos dados são organizados em: expressividade (itens de frequência), críticas (itens de frequência), falar em público (itens de frequência), variáveis de contexto (diversidade na emissão de respostas), comportamentos habilidosos, comportamentos não habilidosos, consequência e consequência negativa. Os respondentes devem responder primeiro aos itens de frequência, e para aqueles que ocorrem frequentemente ou algumas vezes, deve-se responder aos itens de conteúdo, em que o universitário assinala apenas aqueles que lhe parecerem verdadeiros. O alpha do instrumento foi de 0,953 na amostra de origem e de 0,960 nessa amostra.

Inventário de Depressão de Beck (BDI). Foi incluído no estudo como uma medida de saúde mental, enquanto um rastreador de indicadores de depressão. O instrumento foi traduzido, adaptado e validado para a população brasileira por Cunha (2001), é autoadministrado, composto por 21 itens que avaliam a gravidade dos sintomas de depressão, os quais são pontuados em uma escala likert de 0 (absolutamente não) a 3 (gravemente), permite classificações da depressão nos níveis mínimo (0-11), leve (12-19), moderado (20-35) e grave (36-63). Originalmente criado para uso com pacientes psiquiátricos, esse instrumento também se mostrou adequado para o uso na população geral. Na população de origem o alpha foi de 0,89 e nessa amostra de 0,872.

Versão reduzida do Inventário de Fobia Social - Mini-SPIN. Trata-se de um instrumento que avalia indicadores diagnósticos de fobia social, e foi incluído no estudo como uma medida de saúde mental, relativa a ansiedade. É composto por três dos 17 itens do instrumento original Social Phobia Inventory (SPIN), no modelo 
checklist, tipo Likert de cinco pontos, com variação quanto à afirmativa do item, de "nada" a "extremamente". Proposto por Connor, Kobak, Churchill, Katzelnick \& Davidson, (2001) inclui os itens 6,9 e 15 ( 6 - o medo de ficar constrangido me faz evitar fazer coisas ou falar com outras pessoas, 9 - eu evito atividades nas quais sou o centro das atenções e 15 - ficar constrangido ou parecer estúpido estão entre meus piores medos), por serem aqueles que em estudo empírico se mostraram como os mais discriminativos de pessoas com transtorno de ansiedade social. No estudo original, a nota de corte 6 apresentou excelentes indicadores de sensibilidade $(88,7 \%)$ e de especificidade (90\%). No Brasil o instrumento foi traduzido e adaptado por Osório (2008), com bons indicadores psicométricos. Tendo apresentado valores de correlação de 0,88 $(p<0,01)$ com a forma completa do SPIN, fidedignidade pelo alfa de Cronbach de 0,73 e bons indicadores de validade discriminativa na comparação com a SCID, com área sob a curva de $0,81(p<0,01)$, erro padrão de 0,02, com intervalo de confiança de $95 \%$, e ainda, com a nota de corte 6 apresentou sensibilidade de 0,94 e especificidade de 0,46. Na presente a amostra o alpha foi de 0,890.

Entrevista clínica estruturada para o DSM-IV (SCID-I - versão clínica - Del-Ben et al., 2001): foi incluída no estudo, por ser considerada uma medida de saúde mental que permite a classificação diagnóstica por transtornos, assim como a classificação de ausência de transtorno psiquiátrico, tratando-se de um instrumento diagnóstico, considerado padrão ouro em estudos clínicos. Proposta por First, Spitzer, Gibon \& Willians (1997), traduzida e adaptada para o português por Del-Ben et al. (2001) também possui satisfatórias propriedades psicométricas. Os aplicadores foram treinados por profissional experiente com 0 instrumento de outra instituição de ensino superior que possui parceria em grupo de pesquisa. A confiabilidade Kappa para o estudo de Del-Ben et al. (2001) foi de 0,87 para transtornos de humor. $O$ Alpha para o presente estudo foi de 0,85 .

\subsection{Procedimentos de coleta de dados}

A coleta dos dados foi coletiva, ou seja, realizada em salas de aula com a presença da turma, mas com o preenchimento individual, após autorização das Coordenações de Cursos e dos respectivos professores que foram contatados em salas de aula, momentos em que foram oferecidos os devidos esclarecimentos sobre os objetivos da mesma. Os participantes que concordaram em participar da pesquisa assinaram um Termo de Consentimento Livre e Esclarecido e receberam uma carta explicativa sobre os objetivos do projeto e um caderno contendo instruções sobre a aplicação dos instrumentos acima descritos, com exceção da SCID, que foi conduzida por 
telefone posteriormente. A amostra total foi constituída por 1346, dos quais 609 universitários também foram entrevistados por telefone na aplicação da SCID. Para a presente pesquisa foram selecionados os participantes que apresentavam indicadores de depressão pelo BDI e/ou diagnóstico pela SCID, conforme critérios abaixo descritos.

O Grupo de Depressão é composto, então, por 64 participantes (24 homens e 40 mulheres). Desses universitários, 35 responderam tanto - BDI quanto a SCID e 29 responderam apenas ao BDI. Dos universitários que responderam aos dois instrumentos, 11 pontuaram apenas na SCID depressão e apenas dois deles, em ambos os instrumentos (SCID e BDI). Dos 64 participantes, 31 deles também pontuaram na Mini-Spin (indicador de fobia social), mas não foram excluídos da amostra.

Do grupo não clínico, composto por 413 universitários, que não pontuaram em qualquer dos instrumentos de saúde mental utilizados, foram selecionados e pareados $64 \mathrm{com}$ as características do grupo clínico quanto ao tipo de curso e sexo. Nota-se que foi possível parear 61 participantes não clínicos considerando ambas as variáveis (sexo e curso), os demais três participantes foram pareados apenas quanto a sexo, de forma que a amostra ficou constituída por 24 homens clínicos e 24 homens não clínicos e por 40 mulheres clínicas e 40 não clínicas.

\subsection{Critérios de inclusão e exclusão}

Foram incluídos na amostra os participantes que apresentaram indicativo de depressão no BDI e/ou diagnóstico na SCID. Universitários que também apresentaram diagnóstico de Fobia Social em comorbidade com indicativo de depressão ou diagnóstico de depressão não foram excluídos da amostra.

\subsection{Procedimentos de tratamento e análise de dados}

O grupo clínico foi comparado com um grupo não clínico utilizando o teste $t$. Os resultados estão apresentados na forma de tabelas. Foi considerada a significância de p menor ou igual a 0,05.

\section{Resultados}

A seção de resultados está organizada de forma a descrever, por interlocutor (mãe, pai, irmãos, amigos, colegas e namorado) características das interações sociais estabelecidas (sentimentos e consequências) na comparação entre o grupo clínico e não clínico. As médias, desvio padrão, valor de $\mathrm{t}$ e significância estatística 
encontram-se descritos nas tabelas abaixo. Foram omitidas as comparações que não diferenciaram os grupos clínico e não clínico.

\section{Interlocutor: MÃE}

Tabela 1 - Descrição das características das interações sociais estabelecidas (sentimento e consequências) na comparação entre o grupo clínico e não clínico para o interlocutor mãe.

\begin{tabular}{|c|c|c|c|}
\hline & $\begin{array}{c}\text { Clínico } \\
\text { Média (DP) }\end{array}$ & $\begin{array}{l}\text { Não clínico } \\
\text { Média (DP) }\end{array}$ & $t^{*}$ \\
\hline \multicolumn{4}{|c|}{ Conversação/perguntas } \\
\hline Demonstra interesse: consequência positiva & $0,63(0,49)$ & $0,83(0,38)$ & $-2,63 * *$ \\
\hline Emite opiniões: consequência positiva & $0,66(0,48)$ & $0,86(0,35)$ & $-2,74 * *$ \\
\hline $\begin{array}{l}\text { Apenas ouve, não fala nada: consequência } \\
\text { negativa }\end{array}$ & $0,17(0,38)$ & $0,03(0,18)$ & $2,69 * *$ \\
\hline Contente, feliz: sentimento positivo & $0,63(0,49)$ & $0,91(0,29)$ & $-3,95^{* *}$ \\
\hline Inseguro: sentimento negativo & $0,28(0,45)$ & $0,13(0,33)$ & $2,22 *$ \\
\hline Despreparado/incapaz: sentimento negativo & $0,17(0,38)$ & $0,05(0,21)$ & $2,29 *$ \\
\hline Ansioso: sentimento negativo & $0,33(0,47)$ & $0,17(0,38)$ & $2,06 *$ \\
\hline $\begin{array}{l}\text { Medo de reprovação dos colegas: sentimento } \\
\text { negativo }\end{array}$ & $0,22(0,42)$ & $0,03(0,18)$ & $3,32 * *$ \\
\hline Medo de reprovação: sentimento negativo & $0,33(0,47)$ & $0,08(0,27)$ & $3,67 * *$ \\
\hline \multicolumn{4}{|c|}{ Sentimento positivo } \\
\hline $\begin{array}{l}\text { Retribui da mesma forma: consequência } \\
\text { positiva }\end{array}$ & $0,80(0,41)$ & $0,94(0,24)$ & $-2,38^{*}$ \\
\hline Contente, feliz: sentimento positivo & $0,69(0,47)$ & $0,91(0,29)$ & $-3,17 * *$ \\
\hline Inseguro: sentimento negativo & $0,19(0,39)$ & $0,06(0,24)$ & $2,16^{*}$ \\
\hline Despreparado/incapaz: sentimento negativo & $0,11(0,31)$ & $0,00(0,00)$ & $2,78 * *$ \\
\hline Satisfeito: sentimento positivo & $0,33(0,47)$ & $0,58(0,50)$ & $-2,91 * *$ \\
\hline \multicolumn{4}{|c|}{ Sentimento negativo } \\
\hline $\begin{array}{l}\text { Percebe, mas espera a outra pessoa vir } \\
\text { conversar: consequência positiva }\end{array}$ & $0,20(0,41)$ & $0,39(0,49)$ & $-2,35^{*}$ \\
\hline $\begin{array}{l}\text { Diz que você não gosta dele: consequência } \\
\text { negativa }\end{array}$ & $0,09(0,29)$ & $0,02(0,13)$ & $1,96 * *$ \\
\hline Fica agressivo: consequência negativa & $0,16(0,37)$ & $0,03(0,18)$ & $2,46^{*}$ \\
\hline Inseguro: sentimento negativo & $0,36(0,48)$ & $0,20(0,41)$ & $1,98^{*}$ \\
\hline $\begin{array}{l}\text { Medo de reprovação de colegas: sentimento } \\
\text { negativo }\end{array}$ & $0,08(0,27)$ & $0,00(0,00)$ & $2,31 *$ \\
\hline Medo de reprovação: sentimento negativo & $0,20(0,41)$ & $0,00(0,00)$ & $4,01 * *$ \\
\hline \multicolumn{4}{|c|}{ Opinião } \\
\hline Despreparado/incapaz: sentimento negativo & $0,13(0,33)$ & $0,03(0,18)$ & $1,99^{*}$ \\
\hline \multicolumn{4}{|c|}{ Fazer críticas } \\
\hline Despreparado/incapaz: sentimento negativo & $0,13(0,33)$ & $0,03(0,18)$ & $1,99^{*}$ \\
\hline \multicolumn{4}{|c|}{ Receber críticas } \\
\hline $\begin{array}{l}\text { Medo das pessoas não prestarem atenção: } \\
\text { sentimento negativo }\end{array}$ & $0,11(0,31)$ & $0,00(0,00)$ & $2,78^{* *}$ \\
\hline Medo de reprovação: sentimento negativo & $0,20(0,41)$ & $0,08(0,27)$ & $2,05^{*}$ \\
\hline
\end{tabular}

${ }^{*} p \leq 0,05,{ }^{* *} p \leq 0,01$. Na tabela estão expressos apenas os resultados com significância estatística.

Quanto às interações com o interlocutor mãe, para conversação/perguntas e expressar sentimentos negativos, o grupo 
clínico relatou mais consequência negativa e sentimento negativo, e o não clínico mais consequência positiva. Quanto à expressão de sentimentos positivos, o grupo clínico descreve ter mais sentimento negativo e o não clínico mais consequência positiva e sentimento positivo. Para opiniões, fazer e receber críticas, o grupo clínico expos mais sentimentos negativos.

\section{Interlocutor: PAI}

Tabela 2 - Descrição das características das interações sociais estabelecidas (sentimento e consequências) na comparação entre o grupo clínico e não clínico para o interlocutor pai.

\begin{tabular}{|c|c|c|c|}
\hline & $\begin{array}{c}\text { Clínico } \\
\text { Média (DP) } \\
\end{array}$ & $\begin{array}{l}\text { Não clínico } \\
\text { Média (DP) } \\
\end{array}$ & $t$ \\
\hline \multicolumn{4}{|c|}{ Conversação/pergunta } \\
\hline $\begin{array}{l}\text { Demonstra interesse: conseqüência } \\
\text { positiva }\end{array}$ & $0,41(0,50)$ & $0,61(0,49)$ & $-2,33^{*}$ \\
\hline $\begin{array}{l}\text { Conversa mostrando desinteresse: } \\
\text { conseqüência negativa }\end{array}$ & $0,30(0,46)$ & $0,13(0,33)$ & $2,42 *$ \\
\hline Contente,feliz: sentimento positivo & $0,34(0,48)$ & $0,59(0,50)$ & $-2,90 * *$ \\
\hline Inseguro: sentimento negativo & $0,38(0,49)$ & $0,14(0,35)$ & $3,12 * *$ \\
\hline $\begin{array}{l}\text { Medo de reprovação: sentimento } \\
\text { negativo }\end{array}$ & $0,28(0,45)$ & $0,09(0,29)$ & $2,77 * *$ \\
\hline \multicolumn{4}{|c|}{ Sentimento positivo } \\
\hline $\begin{array}{l}\text { Retribui da mesma forma: consequência } \\
\text { positiva }\end{array}$ & $0,45(0,50)$ & $0,66(0,48)$ & $-2,34^{*}$ \\
\hline $\begin{array}{l}\text { Demonstra timidez: consequência } \\
\text { negativa }\end{array}$ & $0,38(0,49)$ & $0,17(0,38)$ & $2,68 * *$ \\
\hline Contente, feliz: sentimento positivo & $0,45(0,50)$ & $0,69(0,47)$ & $-2,74 * *$ \\
\hline Inseguro: sentimento negativo & $0,31(0,47)$ & $0,05(0,21)$ & $4,14 * *$ \\
\hline $\begin{array}{l}\text { Despreparado/incapaz: sentimento } \\
\text { negativo }\end{array}$ & $0,11(0,31)$ & $0,00(0,00)$ & $2,78^{* *}$ \\
\hline Ansioso: sentimento negativo & $0,27(0,45)$ & $0,13(0,33)$ & $2,02 *$ \\
\hline Satisfeito: sentimento positivo & $0,23(0,43)$ & $0,44(0,5)$ & $-2,47 *$ \\
\hline $\begin{array}{l}\text { Medo de reprovação: sentimento } \\
\text { negativo }\end{array}$ & $0,17(0,38)$ & $0,02(0,13)$ & $3,12 * *$ \\
\hline \multicolumn{4}{|c|}{ Sentimento negativo } \\
\hline Fica agressivo: consequência negativa & $0,16(0,37)$ & $0,03(0,18)$ & $2,46^{*}$ \\
\hline Inseguro: sentimento negativo & $0,34(0,48)$ & $0,16(0,37)$ & $2,49^{*}$ \\
\hline $\begin{array}{l}\text { Despreparado/incapaz: sentimento } \\
\text { negativo }\end{array}$ & $0,16(0,37)$ & $0,05(0,21)$ & $2,07 *$ \\
\hline $\begin{array}{l}\text { Medo de reprovação de colegas: } \\
\text { sentimento negativo }\end{array}$ & $0,08(0,27)$ & $0,00(0,00)$ & $2,31^{*}$ \\
\hline $\begin{array}{l}\text { Medo das pessoas não prestarem } \\
\text { atenção: sentimento negativo }\end{array}$ & $0,06(0,24)$ & $0,00(0,00)$ & $2,05^{*}$ \\
\hline $\begin{array}{l}\text { Medo de reprovação: sentimento } \\
\text { negativo }\end{array}$ & $0,14(0,35)$ & $0,00(0,00)$ & $3,21 * *$ \\
\hline \multicolumn{4}{|c|}{ Opinião } \\
\hline Fica agressivo: consequência negativa & $0,23(0,43)$ & $0,08(0,27)$ & $2,47^{*}$ \\
\hline Inseguro: sentimento negativo & $0,25(0,44)$ & $0,09(0,29)$ & $2,38 *$ \\
\hline $\begin{array}{l}\text { Despreparado/incapaz: sentimento } \\
\text { negativo }\end{array}$ & $0,16(0,37)$ & $0,05(0,21)$ & $2,07 *$ \\
\hline
\end{tabular}




\begin{tabular}{|c|c|c|c|}
\hline Fica agressivo: consequência negativa & $0,23(0,43)$ & $0,08(0,27)$ & $2,47^{*}$ \\
\hline Inseguro: sentimento negativo & $0,25(0,44)$ & $0,09(0,29)$ & $2,38^{*}$ \\
\hline $\begin{array}{l}\text { Despreparado/incapaz: sentimento } \\
\text { negativo }\end{array}$ & $0,16(0,37)$ & $0,05(0,21)$ & $2,07 *$ \\
\hline \multicolumn{4}{|c|}{ Receber críticas } \\
\hline $\begin{array}{l}\text { Explica, expressa própria opinião: } \\
\text { consequência positiva }\end{array}$ & $0,41(0,50)$ & $0,58(0,50)$ & $-1,96^{*}$ \\
\hline $\begin{array}{l}\text { Despreparado/incapaz: sentimento } \\
\text { negativo }\end{array}$ & $0,22(0,42)$ & $0,06(0,24)$ & $2,59^{*}$ \\
\hline $\begin{array}{l}\text { Medo das pessoas não prestarem } \\
\text { atenção: sentimento negativo }\end{array}$ & $0,08(0,27)$ & $0,00(0,00)$ & $2,31 *$ \\
\hline $\begin{array}{l}\text { Medo de reprovação: sentimento } \\
\text { negativo }\end{array}$ & $0,20(0,41)$ & $0,06(0,24)$ & $2,38^{*}$ \\
\hline
\end{tabular}

Tratando-se das interações com o interlocutor pai, os comportamentos de conversar/perguntar e expressar sentimento positivo apresentam mais consequência negativa e sentimento negativo para o grupo clínico, e mais consequência positiva e sentimento positivo para o grupo sem depressão. Para as habilidades de expressar sentimento negativo, opinião e fazer críticas o grupo clínico narrou mais consequência negativa e sentimento negativo. Quanto a receber críticas, há mais descrição de sentimento negativo para o grupo clinico e para o não clínico mais consequência positiva.

\section{I nterlocutor: I RMÃO}

Tabela 3 - Descrição das características das interações sociais estabelecidas (sentimento e consequências) na comparação entre 0 grupo clínico e não clínico para o interlocutor irmão.

\begin{tabular}{lccc}
\hline & Clínico & Não clínico & t \\
\cline { 2 - 3 } & Média (DP) & Média (DP) & \\
\hline \multicolumn{4}{c}{ Conversação/perguntas } \\
Conversa mostrando interesse: conseqüência positiva & $0,44(0,5)$ & $0,72(0,45)$ & $-3,33^{* *}$ \\
Contente, feliz: sentimento positivo & $0,45(0,50)$ & $0,84(0,37)$ & $-5,03^{* *}$ \\
Inseguro: sentimento negativo & $0,17(0,38)$ & $0,03(0,18)$ & $2,69^{* *}$ \\
\hline \multicolumn{4}{c}{ Sentimento positivo } \\
\hline Retribui da mesma forma: consequência positiva & $0,59(0,50)$ & $0,84(0,37)$ & $-3,25^{* *}$ \\
Contente, feliz: sentimento positivo & $0,53(0,50)$ & $0,84(0,37)$ & $-4,02^{* *}$ \\
Inseguro: sentimento negativo & $0,23(0,43)$ & $0,03(0,18)$ & $3,52^{* *}$ \\
Seguro: sentimento positivo & $0,30(0,46)$ & $0,52(0,50)$ & $-2,56^{*}$ \\
Satisfeito: sentimento positivo & $0,34(0,48)$ & $0,56(0,50)$ & $-2,53^{*}$ \\
\hline \multicolumn{4}{c}{ Sentimento negativo } \\
\hline Diz que você não gosta dele: consequência negativa & $0,13(0,33)$ & $0,02(0,13)$ & $2,46^{*}$
\end{tabular}


Alessandra Turini Bolsoni-Silva, Bárbara Trevizan Guerra O impacto da depressão para as interações sociais de universitários

Inseguro: sentimento negativo

Medo de reprovação de colegas: sentimento negativo

Medo de reprovação: sentimento negativo

$\begin{array}{lll}0,34(0,48) & 0,14(0,35) & 2,74 * * \\ 0,09(0,29) & 0,02(0,13) & 1,96 * * \\ 0,13(0,33) & 0,02(0,13) & 2,46^{*}\end{array}$

Receber críticas

$0,14(0,35) \quad 0,03(0,18) \quad 2,23^{*}$

Para o interlocutor irmão, conversar/perguntar e expressar sentimento positivo, o grupo com depressão narrou mais sentimento negativo e o grupo não clinico mais consequência positiva e sentimento positivo. Em relação a expressar sentimento negativo, o grupo clínico descreveu mais consequência negativa e sentimento negativo, e para receber críticas mais sentimento negativo.

\section{Interlocutor: AMI GOS}

Tabela 4 - Descrição das características das interações sociais estabelecidas (sentimento e consequências) na comparação entre o grupo clínico e não clínico para o interlocutor amigos.

\begin{tabular}{|c|c|c|c|}
\hline & $\frac{\text { Clínico }}{\text { Média (DP) }}$ & $\frac{\text { Não clínico }}{\text { Média (DP) }}$ & $\mathrm{T}$ \\
\hline \multicolumn{4}{|c|}{ Conversação/perguntas } \\
\hline $\begin{array}{l}\text { Demonstra interesse: } \\
\text { conseqüência positiva }\end{array}$ & $0,70(0,46)$ & $0,89(0,31)$ & $-2,69 * *$ \\
\hline $\begin{array}{l}\text { Conversa mostrando interesse: } \\
\text { consequência positiva }\end{array}$ & $0,67(0,47)$ & $0,86(0,35)$ & $-2,55^{*}$ \\
\hline $\begin{array}{l}\text { Contente, feliz: sentimento } \\
\text { positivo }\end{array}$ & $0,70(0,46)$ & $0,92(0,27)$ & $-3,28 * *$ \\
\hline Inseguro: sentimento negativo & $0,28(0,45)$ & $0,09(0,29)$ & $2,78 * *$ \\
\hline Satisfeito: sentimento positivo & $0,41(0,50)$ & $0,61(0,49)$ & $-2,33 *$ \\
\hline $\begin{array}{l}\text { Medo de reprovação de colegas: } \\
\text { sentimento negativo }\end{array}$ & $0,27(0,45)$ & $0,05(0,21)$ & $3,55^{* *}$ \\
\hline $\begin{array}{l}\text { Medo das pessoas não prestarem } \\
\text { atenção: sentimento negativo }\end{array}$ & $0,22(0,42)$ & $0,02(0,13)$ & $3,74 * *$ \\
\hline $\begin{array}{l}\text { Medo de reprovação: sentimento } \\
\text { negativo }\end{array}$ & $0,17(0,38)$ & $0,02(0,13)$ & $3,12 * *$ \\
\hline \multicolumn{4}{|c|}{ Sentimento positivo } \\
\hline $\begin{array}{l}\text { Retribui da mesma forma: } \\
\text { consequência positiva }\end{array}$ & $0,75(0,44)$ & $0,95(0,21)$ & $-3,35^{* *}$ \\
\hline $\begin{array}{l}\text { Contente,feliz: sentimento } \\
\text { positivo }\end{array}$ & $0,73(0,45)$ & $0,91(0,29)$ & $-2,58 *$ \\
\hline Inseguro: sentimento negativo & $0,27(0,45)$ & $0,08(0,27)$ & $2,88^{* *}$ \\
\hline Ansioso: sentimento negativo & $0,22(0,42)$ & $0,08(0,27)$ & $2,27^{*}$ \\
\hline $\begin{array}{l}\text { Medo de reprovação dos colegas: } \\
\text { sentimento negativo }\end{array}$ & $0,09(0,29)$ & $0,00(0,00)$ & $2,55^{*}$ \\
\hline $\begin{array}{l}\text { Medo das pessoas não prestarem } \\
\text { atenção: sentimento negativo }\end{array}$ & $0,11(0,31)$ & $0,02(0,13)$ & $2,22 *$ \\
\hline $\begin{array}{l}\text { Medo de reprovação: sentimento } \\
\text { negativo }\end{array}$ & $0,14(0,35)$ & $0,00(0,00)$ & $3,21 * *$ \\
\hline
\end{tabular}




\begin{tabular}{|c|c|c|c|}
\hline \\
\hline Inseguro: sentimento negativo & $0,39(0,49)$ & $0,22(0,42)$ & $2,13^{*}$ \\
\hline $\begin{array}{l}\text { Medo de reprovação de colegas: } \\
\text { sentimento negativo }\end{array}$ & $0,16(0,37)$ & $0,03(0,18)$ & $2,46^{*}$ \\
\hline $\begin{array}{l}\text { Medo das pessoas não prestarem } \\
\text { atenção: sentimento negativo }\end{array}$ & $0,14(0,35)$ & $0,02(0,13)$ & $2,69 * *$ \\
\hline \multicolumn{4}{|c|}{ Opiniões } \\
\hline $\begin{array}{l}\text { Medo de reprovação de colegas: } \\
\text { sentimento negativo }\end{array}$ & $0,14(0,35)$ & $0,03(0,18)$ & $2,23^{*}$ \\
\hline \multicolumn{4}{|c|}{ Fazer críticas } \\
\hline $\begin{array}{l}\text { Medo de reprovação de colegas: } \\
\text { sentimento negativo }\end{array}$ & $0,14(0,35)$ & $0,03(0,18)$ & $2,23^{*}$ \\
\hline \multicolumn{4}{|c|}{ Receber críticas } \\
\hline $\begin{array}{l}\text { Não diz nada: consequência } \\
\text { negativa }\end{array}$ & $0,25(0,44)$ & $0,06(0,24)$ & $3,00 * *$ \\
\hline Inseguro: sentimento negativo & $0,27(0,45)$ & $0,13(0,33)$ & $2,02 *$ \\
\hline $\begin{array}{l}\text { Medo das pessoas não prestarem } \\
\text { atenção: sentimento negativo }\end{array}$ & $0,13(0,34)$ & $0,00(0,00)$ & $2,36^{*}$ \\
\hline
\end{tabular}

Nas interações com os amigos, quanto às habilidades de conversar/perguntar e expressar sentimento positivo, o grupo clínico expos ter mais sentimento negativo e o não clinico mais consequência positiva e sentimento positivo. Nas situações em que expressa sentimento negativo opinião e faz críticas o grupo com depressão narrou mais sentimento negativo, e para receber críticas mais consequência negativa e sentimentos negativos.

\section{Interlocutor: COLEGAS DE REPÚBLICA}

Tabela 5 - Descrição das características das interações sociais estabelecidas (sentimento e consequências) na comparação entre 0 grupo clínico e não clínico para o interlocutor colegas de república.

\begin{tabular}{lccc}
\hline & Clínico & Não clínico & \multirow{2}{*}{ t* $^{*}$} \\
\cline { 2 - 3 } & Média (DP) & Média (DP) & \\
\hline $\begin{array}{l}\text { Conversa mostrando desinteresse: } \\
\text { consequência negativa }\end{array}$ & $0,16(0,37)$ & $0,03(0,18)$ & $2,46^{*}$ \\
$\begin{array}{l}\text { Medo de reprovação de colegas: } \\
\text { sentimento negativo }\end{array}$ & $0,14(0,35)$ & $0,02(0,13)$ & $2,69^{* *}$ \\
\hline & Sentimento positivo & & \\
\hline $\begin{array}{l}\text { Medo de reprovação de colegas: } \\
\text { sentimento negativo }\end{array}$ & $0,09(0,29)$ & $0(0)$ & $2,55^{*}$ \\
$\begin{array}{l}\text { Medo de reprovação: sentimento } \\
\text { negativo }\end{array}$ & $0,09(0,29)$ & $0(0)$ & $2,55^{*}$ \\
\hline $\begin{array}{l}\text { Fica agressivo: consequência } \\
\text { negativa }\end{array}$ & $0,08(0,27)$ & $0,00(0,00)$ & $2,31^{*}$ \\
\hline $\begin{array}{l}\text { Sai da situação: consequência } \\
\text { negativa }\end{array}$ & Receber críticas & & \\
$\begin{array}{l}\text { Medo de reprovação de colegas: } \\
\text { sentimento negativo }\end{array}$ & $0,08(0,27)$ & $0,00(0,00)$ & $2,31^{*}$ \\
\hline
\end{tabular}


Quanto aos colegas de república, o grupo clínico relatou ter mais consequência negativa e sentimento negativo para conversar/perguntar e receber críticas, mais sentimento negativo no momento em que expressa sentimento positivo e mais consequência negativa quanto faz críticas.

\section{I nterlocutor: NAMORADO}

Tabela 6 - Descrição das características das interações sociais estabelecidas (sentimento e consequências) na comparação entre o grupo clínico e não clínico para o interlocutor namorado.

\begin{tabular}{|c|c|c|c|}
\hline & $\begin{array}{c}\text { Clínico } \\
\text { Média (DP) }\end{array}$ & $\begin{array}{l}\text { Não clínico } \\
\text { Média (DP) }\end{array}$ & $t^{*}$ \\
\hline \multicolumn{4}{|c|}{ Conversação/perguntas } \\
\hline $\begin{array}{l}\text { Demonstra interesse: consequência } \\
\text { positiva }\end{array}$ & $0,34(0,48)$ & $0,52(0,50)$ & $-1,98^{*}$ \\
\hline $\begin{array}{l}\text { Introduz novos assuntos: consequência } \\
\text { positiva }\end{array}$ & $0,33(0,47)$ & $0,5(0,50)$ & $-1,99^{*}$ \\
\hline $\begin{array}{l}\text { Conversa mostrando interesse: } \\
\text { consequência positiva }\end{array}$ & $0,31(0,47)$ & $0,53(0,50)$ & $-2,55^{*}$ \\
\hline Contente, feliz: sentimento positivo & $0,31(0,47)$ & $0,52(0,50)$ & $-2,37^{*}$ \\
\hline $\begin{array}{l}\text { Despreparado/incapaz: sentimento } \\
\text { negativo }\end{array}$ & $0,14(0,35)$ & $0,02(0,13)$ & $2,69 * *$ \\
\hline Seguro: sentimento positivo & $0,19(0,39)$ & $0,47(0,50)$ & $-3,52 * *$ \\
\hline Satisfeito: sentimento positivo & $0,19(0,39)$ & $0,44(0,5)$ & $-3,14^{* *}$ \\
\hline $\begin{array}{l}\text { Medo de reprovação: sentimento } \\
\text { negativo }\end{array}$ & $0,16(0,37)$ & $0,03(0,18)$ & $2,46^{*}$ \\
\hline \multicolumn{4}{|c|}{ Sentimento positivo } \\
\hline $\begin{array}{l}\text { Retribui da mesma forma: consequência } \\
\text { positiva }\end{array}$ & $0,36(0,48)$ & $0,55(0,50)$ & $-2,15^{*}$ \\
\hline Não retribui: consequência negativa & $0,09(0,29)$ & $0,00(0,00)$ & $2,55^{*}$ \\
\hline Seguro: sentimento positivo & $0,17(0,38)$ & $0,39(0,49)$ & $-2,82 * *$ \\
\hline Satisfeito: sentimento positivo & $0,17(0,38)$ & $0,39(0,49)$ & $-2,82^{* *}$ \\
\hline $\begin{array}{l}\text { Medo de reprovação: sentimento } \\
\text { negativo }\end{array}$ & $0,11(0,31)$ & $0,00(0,00)$ & $2,78 * *$ \\
\hline \multicolumn{4}{|c|}{ Sentimento negativo } \\
\hline $\begin{array}{l}\text { Medo de reprovação: sentimento } \\
\text { negativo }\end{array}$ & $0,17(0,38)$ & $0,03(0,18)$ & $2,69^{* *}$ \\
\hline \multicolumn{4}{|c|}{ Opinião } \\
\hline Fica agressivo: consequência negativa & $0,13(0,33)$ & $0,03(0,18)$ & $1,99^{*}$ \\
\hline Seguro: sentimento positivo & $0,08(0,27)$ & $0,25(0,44)$ & $-2,68 * *$ \\
\hline $\begin{array}{l}\text { Medo de reprovação: sentimento } \\
\text { negativo }\end{array}$ & $0,11(0,31)$ & $0,02(0,13)$ & $2,22 *$ \\
\hline \multicolumn{4}{|c|}{ Fazer críticas } \\
\hline Inseguro: sentimento negativo & $0,20(0,41)$ & $0,08(0,27)$ & $2,05^{*}$ \\
\hline Seguro: sentimento positivo & $0,06(0,24)$ & $0,19(0,39)$ & $-2,16^{*}$ \\
\hline $\begin{array}{l}\text { Medo das pessoas não prestarem atenção: } \\
\text { sentimento negativo }\end{array}$ & $0,06(0,24)$ & $0,00(0,00)$ & $2,05^{*}$ \\
\hline \multicolumn{4}{|c|}{ Receber críticas } \\
\hline Satisfeito: sentimento positivo & $0,05(0,21)$ & $0,16(0,37)$ & $-2,07 *$ \\
\hline
\end{tabular}


Por fim, tratando-se das interações com parceiros amorosos, quando conversam/perguntam, o grupo clínico relata ter mais sentimento negativo e o não clínico mais consequência positiva e sentimento positivo. Ao expressar sentimento positivo, o grupo com depressão afirmou encontrar mais consequência negativa e sentimento negativo, e o não clínico mais consequência positiva e sentimento positivo. Nas situações em que expressam sentimentos negativos, 0 grupo clínico relatou ter mais sentimento negativo.

Para as opiniões, o grupo clínico descreveu mais consequência negativa e sentimento negativo, enquanto $o$ não clínico mais sentimento positivo. Ao fazer críticas, os universitários com depressão relataram mais sentimento negativo e o não clínico mais sentimento positivo, e para receber críticas o grupo não clínico afirmou ter mais sentimento positivo.

\section{Discussão}

Os resultados deste estudo indicam, de maneira geral, que os universitários clínicos para depressão apresentam menor repertório de habilidades sociais, relatando obter consequências negativas e sentindo-se mal com maior frequência que os universitários não clínicos.

Quando o universitário clínico para depressão conversa/faz perguntas, obtém como subproduto da interação com todos os interlocutores sentimentos negativos, como sentir-se inseguro, despreparado/incapaz e ansioso. Ainda nessas situações, os pais e colegas de república apresentam consequências negativas quando o estudante conversa, ao demonstrar desinteresse e/ou apenas ouvir e não dizer nada, não incentivando a continuidade da comunicação. Por outro lado, o grupo não clínico relata que os pais demonstram interesse e/ou emitem opiniões nesses momentos e, adicionalmente, sentem-se contentes.

A presença desses sentimentos, associado ao desinteresse dos interlocutores, sinaliza o estabelecimento de contingências aversivas (Abib, 2001), ou seja, quando o universitário conversa com as pessoas, possivelmente elas não dão atenção ou reagem negativamente à sua resposta, o que probabilisticamente reduz a frequência da emissão dessa resposta e tem sentimentos negativos associados, o que realmente parece ocorrer com a amostra clínica, uma vez que apresentam déficits de habilidades sociais e sentimentos negativos. Quanto à expressão de sentimentos positivos, algo semelhante ocorre, o grupo clínico relata sentimentos negativos, tais como insegurança e despreparo/incapacidade, sendo que o pai demonstra timidez e os namorados não retribuem nesses momentos, 
enquanto que no grupo não clínico, os pais retribuem o afeto, deixando-o contente e satisfeito.

Habilidades sociais que envolvem maior asserção, como o expressar sentimento negativo parece trazer estimulações aversivas importantes para o grupo clínico, momentos em que os pais e os irmãos dizem não gostar do filho e/ou agem de forma agressiva, repetidamente os sentimentos de insegurança e medo aparecem com maior frequência. O grupo não clínico parece ter mais repertório ao expressar tais sentimentos, pois a mãe conversa nessas ocasiões, sem agressividade. Quanto a opiniões, fazer e receber críticas os universitários clínicos para depressão relatam sentirem-se despreparados e com medo, principalmente nas interações com pais e amigos, momentos que o pai fica agressivo, os amigos não dizem nada, os colegas de república saem da situação e os namorados ficam agressivos.

Conclui-se, então, que os universitários clínicos relatam interações com amigos, colegas ou namorados que produzem pouco reforçadores, sendo os resultados obtidos muito semelhantes aos encontrados com os familiares. Ao contrário, o grupo não clínico parece se sair bem nessas interações, tal como no convívio com os familiares, pois conseguem obter atenção e interesse do interlocutor, mantem a comunicação e têm sentimentos positivos associados. Chama a atenção que com os colegas de república os grupos não são diferentes para expressar sentimento negativos e opiniões.

Pode-se concluir que os universitários com depressão apresentam uma grande dificuldade quanto ao repertório de habilidades sociais independente das que exigem mais ou menos asserção e de maneira generalizada para todas as interações sociais. Dessa forma, os universitários clínicos parecem obter poucos reforçadores nas interações sociais (Ferster, 1973), justificando o quadro de depressão que se apresenta.

As dificuldades encontradas, no âmbito das habilidades sociais, estão na direção da literatura da área, como lidar com o relacionamento com namorados e familiares, expressar sentimentos positivo, negativo e opinião, lidar com críticas e comunicar-se (Bandeira et al., 2006; Bolsoni-Silva et al., 2010; Del Prette et al., 2004; Ribeiro \& Bolsoni-Silva, 2011).

É certo que a depressão é multideterminada (Vasconcellos, Rocha \& Maciel, 2010) e múltiplas variáveis podem estar associadas a problemas de saúde mental, tais como o abandono escolar (Backer, 2003; Ciarrochi et al., 2002; Cole, Lazarick, \& Howard, 1986; Furtado, Falcone, \& Clark, 2003), variáveis sócio-demográficas (Adlaf et al., 2001; Cerchiari et al., 2005; Neves \& Dalgalarrondo, 2007), de curso (Fang et. al., 2010) e história prévia (Fioretti et al., 2010).

Consequentemente, não se pode afirmar que os desafios da universidade e o repertório de habilidades sociais sejam as causas 
únicas para o surgimento de problemas de saúde mental. No entanto, os resultados da presente pesquisa atestam achados anteriores da literatura (Cole et al., 1986; Ciarrochi et al., 2002; Backer, 2003; Furtado, Falcone \& Clark, 2003), ao verificar que o repertório de habilidades sociais têm impacto para a depressão. Todavia, destacase que o grupo clínico tem reservas comportamentais, ou seja, habilidades sociais utilizadas nas interações sociais avaliadas, ainda que com baixa frequência, uma vez que estão descritas as comparações que apresentaram diferenças estatísticas entre os grupos, e o restante dos itens avaliados no instrumento não diferenciaram os grupos.

Ferster (1973) já afirmava que seria útil identificar grupos clínicos e não clínicos para depressão para descrever padrões comportamentais, o que foi especialmente importante na presente pesquisa. A partir de análises das interações sociais (Goldiamond, 1974/2002), foi possível identificar os comportamentos operantes de habilidades sociais, ou seja, as respostas emitidas pelos estudantes depressivos ou não depressivos, os seus sentimentos e as consequências apresentadas pelos interlocutores.

A partir dos achados da presente pesquisa afirma-se que o treino de habilidades sociais com universitários deprimidos é útil, desde que ensine diversas classes de respostas a serem emitidas com diferentes interlocutores de forma a ter maior probabilidade de gerar consequências reforçadoras e, como subproduto, sentimentos positivos.

As intervenções psicológicas nas universidades são de extrema importância, considerando-se o período de estudo na universidade e os danos que a depressão pode ocasionar quando o estudante se forma, uma vez que o profissional com baixa competência social e comunicação ineficaz, apresenta prejuízos para sua vida profissional (Murakami, Murray, Sims \& Chedzey, 2009). Garantindo a intervenção adequada para a população clínica com transtorno mental, cujo índice é alto em estudantes universitários, problemas futuros poderiam ser prevenidos e remediados, enquanto ainda se é um adulto jovem. No mesmo sentido, Fioretti et al. (2010) ao analisar a alta prevalência de transtornos mentais comuns em universitários, identificam a necessidade de cuidar da saúde mental dos estudantes, visando a qualidade de vida dos mesmos.

Além disso, as intervenções têm encontrado bons resultados, pois segundo Peres et al., (2004), poucos alunos que buscaram por atendimento na universidade interromperam a intervenção antes do desfecho planejado, o que pode ser considerado um indicador positivo da aceitação do serviço por parte da população assistida. Assim, como a adesão ao tratamento é satisfatória, deveria ser estimulada pela universidade, inclusive propondo trabalhos com enfoque preventivo (por exemplo Allan, 1992, Bolsoni-Silva, 2009). 
Outro fator que pode contribuir é a estimulação da universidade em informar e oferecer serviços psicológicos. Thomas, Caputi e Wilson (2014) investigaram atitudes e intenções em estudantes de Psicologia para buscar tratamento para transtornos mentais. Os autores encontraram que os fatores que predizem a busca por ajuda estão relacionados ao reconhecimento de sintomas, benefícios da ajuda profissional e abertura ao tratamento para os problemas emocionais. Logo, se os estudantes tiverem conhecimento sobre os transtornos, sobre como a psicoterapia pode auxiliar, há maior chance de buscarem pela ajuda.

\section{Considerações finais}

Ainda que existam estudos preocupados em avaliar o repertório de habilidades sociais em universitários poucos são aqueles que o fazem na interface com a saúde mental; menos frequentes ainda são as pesquisas que avaliam além da frequência comportamental, o impacto para as interações sociais, no que se refere às consequências e sentimentos relatados. Nesse sentido, a presente pesquisa traz contribuições importantes, ampliando o conhecimento sobre comportamentos de habilidades sociais nas diferentes interações sociais e indicadores de saúde mental para o universitário. Quanto ao cuidado metodológico é importante destacar que o grupo clínico e não clínico foram pareados quanto a curso e sexo, dando maior controle de variáveis.

Como limitações tem-se que parte da amostra apresenta indicadores de depressão e outra, depressão propriamente dita, o que poderia ser controlado em futuros estudos. Ainda, aproximadamente metade da amostra clínica possui indicadores de ansiedade como comorbidade. Outras pesquisas também poderiam isolar variáveis importantes como trabalho e tipo de curso, que são variáveis que podem ter influenciado nos resultados encontrados. Ainda, seria interessante que novos estudos avaliassem a influência de gênero e de universidades particulares, uma vez que a pesquisa foi realizada em uma instituição pública e a maioria desta amostra foi do sexo feminino.

\section{Referências}

Abib, J. A. D. (2001). Teoria moral de Skinner e desenvolvimento humano. Psicologia: Reflexão e Crítica, 14(1), 107-117.

Adlaf, E. M., Gliksman, L., Demers, A. \& Newton-Taylor, B. (2001). The prevalence of elevated psychological distress among Canadian undergraduates: findings from the 1998 Canadian 
Campus Survey. Journal of American College Health, 5(2), 6772.

Allan, M. S. (1992). From student to professional: students perspectives on professional development during clinical psychology training. Dissertation, St. John's University Jamaica: New York.

American Psychiatric Association. (2000). Diagnostic and statistical manual of mental disorders. DSM-IV-TR. 4a. Edição.

Backer, S. R. (2003). A prospective longitudinal investigation of social problem- solving appraisals on adjustment to university, stress, health and academic motivation and performance. Personality and Individual Differences, 35, 569-591.

Banaco, R. A., Zamignani, D. R. \& Meyer, S. B. (2010). Função do Comportamento e do DSM: Terapeutas analíticocomportamentais discutem a psicopatologia. In Tourinho, E. Z. \& Luna, S. V. (Orgs.) Análise do comportamento: Investigações históricas, conceituais e aplicadas. São Paulo: Roca.

Bandeira, M., Rocha, S. S., Freitas, L. C., Del Prette, Z. A. P. \& Del Prette, A. (2006). Habilidades sociais e variáveis sociodemográficas em estudantes do ensino fundamental, Psicologia em Estudo, 11(3), 541-549.

Bandeira, M. \& Quaglia, M.A.C. (2005). Habilidades sociais de estudantes universitários: identificação de situações sociais significativas. Interação, 9(1), 45-55.

Bolsoni-Silva, A. T. (2002). Habilidades sociais: breve análise da teoria e da prática à luz da análise do comportamento. Interação em Psicologia, 6(2), 233-242.

Bolsoni-Silva, A. T. (2009). Avaliação de um treinamento de habilidades sociais (THS) com universitários e recém-formados. Interação em Psicologia, 13(2), 241-251.

Bolsoni-Silva, A. T. (2011). Habilidades sociais e saúde mental de estudantes universitários: Construção e Validação do Q-ACC-VU e Estudos Clínicos em Análise do Comportamento. Tese de Livre-Docência, Faculdade de Ciências, Universidade Estadual Paulista Júlio de Mesquita Filho, Bauru, SP.

Bolsoni-Silva, A. T., Loureiro, S. R., Rosa, C. F. \& Oliveira, M. C. F. A. (2010). Caracterização das habilidades sociais de universitários. Contextos Clínicos, 3(1), 62-75.

Bolsoni-Silva, A. T. \& Carrara, K. (2010). Habilidades Sociais e Análise do Comportamento: compatibilidades e dissensões conceitual-metodológicas. Psicologia em Revista (Online), 16(2), 330-350.

Boas, A. C. V. V., Silveira, F. F. \& Bolsoni-Silva, A. T. (2005). Descrição de efeitos de um procedimento de intervenção em grupo com universitários: um estudo piloto. Interação em Psicologia, 9(2), 323-332. 
Catania, A. C. (1998). Aprendizagem: comportamento, linguagem e cognição. 4. Ed. Porto Alegre: Artes Médicas, 1999.

Cerchiari, E. A. N., Caetano, D. \& Faccenda, O. (2005). Prevalência de transtornos mentais menores em estudantes universitários. Estudos de Psicologia, 10(3), 413-420.

Christensen, P. N., Stein, M. B. \& Means-Christensen, A. (2003). Social anxiety and interpersonal perception: a social relations model analysis. Behaviour Research and Therapy, 41, 13551371.

Ciarrochi, J., Deane, F. P. \& Anderson, S. (2002). Emotional intelligence moderates the relationship between stress and mental health. Personality and Individual Differences, 32, 197209.

Cole, D. A., Lazarick, D. L. \& Howard, G. S. (1986). Construct validity and the relation between depression and social skill. Journal of Counseling Psychology, 34(3), 315-321.

Corchs, F. (2010). É possível ser um psiquiatra behaviorista radical? Primeiras reflexões. Perspectivas em Análise do Comportamento, 1(1), 55-66.

Connor, K. M., Kobak, K. A., Churchill, L. E., Katzelnick, D. \& Davidson, J. R. (2001). Mini-Spin: a brief screening assessment for generalized social anxiety disorder. Depression and Anxiety, $14,137-140$.

Cunha, J. A. (2001). Manual da versão em português das Escalas Beck. São Paulo: Casa do Psicólogo.

Del-Ben, C. M., Vilela, J. A. A., Crippa, J. A. S., Hallak, J. E., Labate, C. M. \& Zuardi, A. W. (2001). Confiabilidade teste-reteste da Entrevista Clínica Estruturada para o DSM-IV (SCID) versão clínica. Revista Brasileira de Psiquiatria, 23(6), 156-159.

Del Prette, A. (1982). Treinamento comportamental em grupo junto à população não clínica de baixa renda: Uma análise descritiva de procedimento. Dissertação (Mestrado em Psicologia Clínica) Pontifícia Universidade Católica de Campinas, Campinas.

Del Prette, A. \& Del Prette, Z. A. P. (2003). No contexto da travessia para 0 ambiente de trabalho: treinamento de habilidades sociais com universitários. Estudos de Psicologia (Natal), 8(3), 413-420.

Del Prette, Z. A. P., Del Prette, A. \& Barreto, M. C. M. (2006). Treinamento de habilidades sociais em grupo com estudantes de Psicologia: Avaliando um programa de intervenção. In: Bandeira, M., Del Prette, Z. A. P., \& Del Prette, A. (Orgs.). Estudos sobre habilidades sociais e relacionamento interpessoal. São Paulo: Casa do Psicólogo.

Del Prette, Z. A. P., Del Prette, A., Barreto, M. C. M., Bandeira, M., Rios-Saldana, M. R., Ulian, A. L. A. O., Gerk-Carneiro, E., Falcone, E. M. O. \& Villa, M. B. (2004). Habilidades sociais de 
estudantes de Psicologia: um estudo multicêntrico. Psicologia: Reflexão e Crítica, 17(3), 341-350.

Delitti, M. \& Meyer, S. B. (1998). O uso dos encobertos na prática da terapia comportamental. In: Rangé, B. (Org.). Psicoterapia comportamental e cognitiva dos transtornos psiquiátricos. Campinas: Editorial Psy II.

Edmondson, C. B., Conger, J. C. \& Conger, A. J. (2007). Social skills in college students with high trait anger. Journal of Social and Clinical Psychology, 26(5), 575-594.

Facundes, V. L. D. \& Ludermir, A. B. (2005). Common mental disorders among health care students. Revista Brasileira de Psiquiatria, 27(3), 194-200.

Fang, D., Young, C., Golshan, S., Fellows, I., Moutier, C. \& Zisook, S. (2010). Depression in Premedical Undergraduates: A CrossSectional Survey Prim Care Companion. Journal of Clinical Psychiatry, 12(6).

Ferster, C. B. (1973). A functional analysis of depression. American Psychology, 28, 857-870.

Fioretti, K. P., Rossoni, R. R., Borges, L. H. \& Miranda, A. E. (2010). Transtornos mentais comuns entre os estudantes do curso de medicina: prevalência e fatores associados. Jornal Brasileiro de Psiquiatria, 59(1), 17-23.

First, M.B., Spitzer, R.L., Gibon, M. \& Willians, J.B.W. (1997). Structured Clinical Interview for DSM-IV Axis I Disorders Clinican Version (SCID-CV). Washington, DC: American Psychiatric Press.

Fitts, S. D., Sebby, R. A. \& Zlokovich, M. S. (2009). Humor styles as mediators of the shyness-loneliness relationship. North American Journal of Psychology, 11(2), 257-272.

Furtado, E. S., Falcone, E. M. O. \& Clark, C. (2003). Avaliação do estresse e das habilidades sociais na experiência acadêmica de estudantes de medicina de uma universidade do Rio de Janeiro. Interação em Psicologia, 7(2), 43-51.

Gerk-Carneiro, E. (2003). Inteligência social como vertente cognitiva da competência social. Em: Del Prette, \& Del Prette (Orgs.). Habilidades sociais, desenvolvimento e aprendizagem: questões conceituais, avaliação e intervenção. Campinas: Alínea.

Goldiamond, I. (1974). Toward a constructional approach to social problems: Ethical and constitucional issues raised by Applied Behavior Analysis. Behavior and Social Issues, 11, 108-197, 2002.

McFall, R. M. (1982). A review and reformulation of the concept of social skills. Behavioral Assessment, 4, 1-33.

McGaha, V. \& Fitzpatrick, J. (2005). Personal and social contributors to dropout risk for undergraduate students. College Student Journal, 39(2), 287-297. 
Murakami, K., Murray, L., Sims, D. \& Chedzey, K. (2009). Learning on placement: the narrative development of social competence. Journal Adult Development, 16, 13-24.

Neves, M. C. C. \& Dalgalarrondo, P. (2007). Transtornos mentais auto-referidos em estudantes universitários. Jornal Brasileiro de Psiquiatria, 56(4), 237-244.

Osório, F. L., Crippa, J. A. S. \& Loureiro, S. R. (2007). A study of the discriminative validity of a screening tool (Mini-Spin) for social anxiety disorders applied to Brazilian university students. European Psychiatry (Paris), 20, 1-5.

Ozben, S. (2013). Social skills, life satisfaction, and loneliness in turkish university students. Social behavior and personality, 41(2), 203-213.

Peres, R. S., Santos, M. A. \& Coelho, H. M. B. (2004). Perfil da clientela de um programa de pronto-atendimento psicológico a estudantes universitários. Psicologia em Estudo, 9(1), 47-54.

Ribeiro, D. \& Bolsoni-Silva, A. T. (2011). Potencialidades e dificuldades interpessoais de universitários: estudo de caracterização. Revista Acta Comportamentalia, 19(2), 205224.

Teixeira, M. A. P., Dias, A. C. G., Wothich, S. H. \& Oliveira, A. M. (2008). Adaptação à universidade em jovens calouros. Psicologia Escolar e Educacional, Campinas, 12(1), 185-202.

Thomas, S. J., Caputi, P. \& Wilson, C. J. (2014). Specific atitudes which predict psychology student's intentios to seek help for psychological distress. Journal of clinical psychology, 70(3), 273-282.

Tourinho, E. Z. (1999). Eventos privados em uma ciência do comportamento. In: Banaco, R. A. (Org.). Sobre comportamento e cognição, Santo André: Arbytes.

Vasconcellos, M., Rocha, M. C. D. O., \& Maciel, V. H. (2010). Revisão teórica sobre depressão pela análise do comportamento e por alguns manuais psiquiátricos. ConScientiae Saúde, 9(4), 719725.

\section{Endereço para correspondência \\ Alessandra Turini Bolsoni-Silva}

Faculdade de Ciências - UNESP - Campus de Bauru

Av. Eng. Luiz Edmundo Carrijo Coube, 14-01, Vargem Limpa, CEP 17033-360, Bauru - SP, Brasil

Endereço eletrônico: bolsoni@fc.unesp.br

\section{Bárbara Trevizan Guerra}

Faculdade de Ciências - UNESP - Campus de Bauru

Av. Eng. Luiz Edmundo Carrijo Coube, 14-01, Vargem Limpa, CEP 17033-360,

Bauru - SP, Brasil

Endereço eletrônico: barbaratrevizan@hotmail.com

Recebido em: 13/01/2014 
Alessandra Turini Bolsoni-Silva, Bárbara Trevizan Guerra O impacto da depressão para as interações sociais de universitários

Reformulado em: 30/03/2014

Aceito para publicação em: 31/03/2014

\section{Notas}

* Psicóloga, Livre Docente e Coordenadora do Programa de Pós-Graduação em Psicologia do Desenvolvimento e Aprendizagem na Unesp Campus Bauru/ Brasil.

** Psicóloga e Mestranda no Programa de Pós-Graduação em Psicologia do Desenvolvimento e Aprendizagem na Unesp Campus Bauru/ Brasil. 